\title{
On Fuzzy Solutions for Diffusion Equation
}

\author{
Jefferson Leite, ${ }^{1}$ Moiseis Cecconello, ${ }^{2}$ Jackellyne Leite, ${ }^{3}$ and R. C. Bassanezi ${ }^{4}$ \\ ${ }^{1}$ Federal University of Piaui, 64049-550 Teresina, PI, Brazil \\ ${ }^{2}$ Federal University of Mato Grosso, 78060-900 Cuiabá, PI, Brazil \\ ${ }^{3}$ Federal Institute of Maranhão, 65635-468 Timon, MA, Brazil \\ ${ }^{4}$ Federal University of ABC, 09210-580 Santo André, SP, Brazil \\ Correspondence should be addressed to Jefferson Leite; jleite@ufpi.edu.br
}

Received 22 July 2014; Accepted 23 November 2014

Academic Editor: T. Warren Liao

Copyright (C) 2015 Jefferson Leite et al. This is an open access article distributed under the Creative Commons Attribution License, which permits unrestricted use, distribution, and reproduction in any medium, provided the original work is properly cited.

Our main goal is to define a fuzzy solution for problems involving diffusion. To this end, the solution of fuzzy diffusion-reactionadvection equation will be defined as Zadeh's extension of deterministic solution of the associated problem. Important aspects such as unity and stability of these solutions will also be studied. Graphical representations of these solutions will be presented.

\section{Introduction}

In 1965, Zadeh introduced the concept of fuzzy sets [1]. Therefore, much research has been developed botkh from a theoretical and practical [2-11]. One area of great interest was the modelling uncertain phenomena by means of differential equations. When subjectivity is random, the nature of uncertainty can be treated as a tool for stochastic differential equations; however, the fuzzy variational models can include various types of uncertainties beyond the stochastic.

Depending on the choice of state variable and/or model parameters, respectively, demographic fuzziness state variables are modelled by fuzzy sets and/or environmental fuzziness only when the parameters are considered fuzzy. In biological phenomena in general, both types are present fuzziness [2].

The fuzzy variational equations have been studied by different methods. The first attempt to include subjectivity of the nonrandom systems was with the variational derivative of Hukuhara $[12,13]$, but this formulation is not able to reproduce the rich behaviour of deterministic differential equations such as periodicity, stability, and bifurcation.

In 1999, [14] proposed another way of looking at subjectivity not by random differential inclusions. The fuzzy solutions obtained by this approach are able to present frequency, stability, and bifurcation; however, from a technical standpoint, to obtain solutions through a family of differential inclusions can be extremely difficult.

When the variables are uncertain, a way to have a more adequate treatment variational models for all uncertainty is to pass parameters to the mathematical model. In this case, an alternative method that is used is that which is to make fuzzy solutions of a deterministic model, using Zadeh's extension [15].

Zadeh's extension is the way we produce a fuzzy transformation $\hat{f}: \mathscr{F}(X) \rightarrow \mathscr{F}(X)$ for a given function $f: X \rightarrow X$. Zadeh's extension $\widehat{f}$ has been studied and applied by many authors, including $[12,16-18]$, in the study of fuzzy fractals and Nguyen [7] in set-representation of fuzzy sets.

Our main objective in this paper is to explore properties such as uniqueness and stability of the fuzzy solution of a fuzzy differential equation associated with classical advection-diffusion-reaction equation (see Figure 9), using Zadeh's extension [19].

\section{Basic Concepts}

2.1. Fuzzy Sets. In this section we present the basic fundamentals necessary for the proper understanding and application of fuzzy set theory in the following sections. For more information, the reader may consult $[2,19]$. The fuzzy subsets 
of a space $X$ are characterized by functions of space in the interval $[0,1]$. Therefore we have the following.

Definition 1. Let $X$ be a nonempty set. A fuzzy subset $F$ of $X$ is a subset $\left\{\left(x, \mu_{F}(x)\right): x \in X\right\}$ nonempty of $X \times[0,1]$ for some function $\mu_{F}: \rightarrow[0,1]$.

The function $\mu_{F}: X \rightarrow[0,1]$ is called the membership function of $F$ and the value assumed for each $x \in X$ is the degree of relevance of $x$ in $F$. The set consists of all fuzzy subsets of a set $X$ that will be denoted by $\mathscr{F}(X)$.

Directly follow that, when defining that any subset random $X$ is also a fuzzy subset of $X$, since this set is well determined by the characteristic function and therefore satisfies the definition above. In fact, if $F$ subset $X$ with characteristic function $\chi_{F}: X \rightarrow[0,1]$, then the set $\chi_{F}=$ $\left\{\left(x, \chi_{F}(x)\right): x \in X\right\}$ is a subset of $X \times[0,1]$ with membership function $\mu_{\chi_{F}}(x)=\chi_{F}(x)$ for all $x \in X$ and hence $\chi_{F} \in \mathscr{F}(X)$. To a better distinction, the fuzzy subset $\chi_{F}$ will be referred to herein as set crisp of $X$.

Given a fuzzy subset $F$ in $\mathscr{F}(X)$ defined, for each $\alpha \epsilon$ $(0,1]$, the set $[F]^{\alpha} \subset X$ is the set of elements of $X$ such that the degree of relevance in $F$ is at least $\alpha$. The set $[F]^{\alpha} \subset X$ is called $\alpha$-level of $F$ and, mathematically, is defined as

$$
[F]^{\alpha}=\left\{x \in X: \mu_{A}(x) \geq \alpha\right\} \quad \text { para } \alpha \in(0,1] .
$$

The zero level of a fuzzy subset $F$ is defined by

$$
[F]^{0}=\overline{\bigcup_{\alpha \in(0,1]}[F]^{\alpha}}=\overline{\operatorname{supp}(F)},
$$

where $\operatorname{supp}(F)=\left\{x \in X: \mu_{F}(x)>0\right\}$ is the support of fuzzy subset $F$.

Two fuzzy sets are equal when the characteristic functions are equal, that is,

$$
A=B \Longleftrightarrow \mu_{A}(x)=\mu_{B}(x) \quad \forall x \in X .
$$

The equality between fuzzy sets can also be characterized through $\alpha$-levels. In this case, the sets are equal when $\alpha$-level matches for every $\alpha \in[0,1]$.

In many cases it may be necessary to extend the domain of a application $f: X \rightarrow Y$ for the fuzzy sets in $\mathscr{F}(X)$. Note that for each subset $F \subset X$ an application $f$ defines a subset $f(F) \subset Y$. Assuming now that $F$ is a fuzzy subset $X$, that is, $F \in \mathscr{F}(X)$, then we need to determine how the image induced the application $f$ over $F$. The way this picture is can be characterized by principle of Zadeh's extension as defined below.

Definition 2. Let $f: X \rightarrow Y$ be a $F$ applying a fuzzy subset $X$. Zadeh's extension $\widehat{f}: \mathscr{F}(X) \rightarrow \mathscr{F}(Y)$ whose image is applied has membership function:

$$
\mu_{\hat{f}(F)}(y)= \begin{cases}\sup _{a \in f^{-1}(y)} \mu_{F}(a) & \text { se } f^{-1}(y) \neq \emptyset \\ 0 & \text { se } f^{-1}(y)=\emptyset\end{cases}
$$

As mentioned earlier, a subset $F \subset X$ determines fuzzy set $\chi_{F}$ of $X$ whose membership function is a characteristic function of $F$. The image of $\chi_{F}$ by extension $\widehat{f}$ that is a function $f$ coincides with the fuzzy set $\chi_{f(F)}$ defined by $f(F)$. That is, $\widehat{f}\left(\chi_{F}\right)=\chi_{f(F)}$. In fact, the above definition ensures that $\widehat{f}\left(\chi_{F}\right)$ has membership function:

$$
\mu_{\widehat{f}\left(\chi_{F}\right)}(y)= \begin{cases}1 & \text { if } y \in f(F), \\ 0 & \text { if } y \in Y-f(F)\end{cases}
$$

which is the characteristic function of $f(F)$. Therefore, $\widehat{f}\left(\chi_{F}\right)=\chi_{f(F)}$. In particular, for all $x \in X$ it is worth $\widehat{f}\left(\chi_{x}\right)=$ $\chi_{\{f(x)\}}$.

Theorem 3. If $f: \mathbb{R}^{n} \rightarrow \mathbb{R}^{n}$ is continuous, then Zadeh's extension $\widehat{f}: \mathscr{F}\left(\mathbb{R}^{n}\right) \rightarrow \mathscr{F}\left(\mathbb{R}^{n}\right)$ is continuous and satisfies

$$
[\widehat{f}(\mathbf{x})]^{\alpha}=f\left([\mathbf{x}]^{\alpha}\right)
$$

for all $\alpha \in[0,1]$.

By the Hausdorff metric $d_{H}$, for the compacts of $X$, we can define a metric for the set $\mathscr{F}(X)$. Thus, given two points $\mathbf{a}, \mathbf{b} \in \mathscr{F}(X)$, the distance $d_{\infty}(\mathbf{a}, \mathbf{b})$ between $\mathbf{a}$ and $\mathbf{b}$ is given by

$$
d_{\infty}(\mathbf{a}, \mathbf{b})=\sup _{\alpha \in[0,1]} d_{H}\left([\mathbf{a}]^{\alpha},[\mathbf{b}]^{\alpha}\right)
$$

where

$$
\begin{aligned}
d_{H} & \left([\mathbf{a}]^{\alpha},[\mathbf{b}]^{\alpha}\right) \\
& =\max \left\{\operatorname{dist}\left([\mathbf{a}]^{\alpha},[\mathbf{b}]^{\alpha}\right), \operatorname{dist}\left([\mathbf{b}]^{\alpha},[\mathbf{a}]^{\alpha}\right)\right\} \\
& =\max \left\{\sup _{a \in[\mathbf{a}]^{\alpha}} \inf _{b \in[\mathbf{b}]^{\alpha}}\|a-b\|, \sup _{b \in[\mathbf{b}]^{\alpha}} \inf _{a \in[\mathbf{a}]^{\alpha}}\|a-b\|\right\} .
\end{aligned}
$$

Particular type of fuzzy set that will prove useful is the call number fuzzy, which is an attempt to generalize real numbers in the fuzzy context.

Definition 4. A fuzzy set $F \subset \mathbb{R}$ is called fuzzy number if satisfies the following:

(a) the $\alpha$-level $[F]^{\alpha}$ are compact, connected, and nonempty for all $\alpha \in[0,1]$;

(b) there is a unique $x$ such that $\mu_{F}(x)=1$.

Definition 5. Let $[u(x, t)]^{\alpha}$ be the $\alpha$-level associated with a function fuzzy $\widehat{u}(x, t)$ and $u_{1}^{\alpha}(x, t), u_{2}^{\alpha}(x, t)$ functions such that

$$
[u(x, t)]^{\alpha}=\left[u_{1}^{\alpha}(x, t), u_{2}^{\alpha}(x, t)\right]
$$

and then define a diameter of $[u(x, t)]^{\alpha}$ as

$$
\operatorname{diam}\left([u(x, t)]^{\alpha}\right)=u_{2}^{0}(x, t)-u_{1}^{0}(x, t) .
$$




\subsection{Diffusion Equation}

2.2.1. Diffusion-Reaction-Advection Equation in $\mathbb{R}$ and $\mathbb{R}^{2}$. Diffusion models have been extensively employed to investigate dispersal and have yielded considerable insight into the dynamics of animal movement in space and time. Diffusion models can be written in the simplest form as

$$
\frac{\partial u}{\partial t}=D \nabla^{2} u+f\left(u, \frac{\partial u}{\partial x}\right)
$$

where the operator $\nabla$ denotes the spatial gradient, $t$ is time, $u(x, y, t)$ is the local population density in the spatial variables $x$ and $y, D$ is the coefficient of diffusion, and $f(u, \partial u / \partial x)$ is the reaction-advection term describing the net population change due to birth, death, and direction of travel.

Most of the phenomena involving diffusion are described by models that suggest a dynamic in $\mathbb{R}$ and $\mathbb{R}^{2}$ is what will be treated below. Consider the initial value problem given by

$$
\begin{gathered}
\frac{\partial u}{\partial t}(x, t)=D \Delta(u(x, t)), \quad \text { in } x \in \mathbb{R}, t \geq 0, \\
u(x, 0)=N_{0} \delta(x), \quad \text { on } \mathbb{R} \times\{t=0\},
\end{gathered}
$$

where $u: U \subset \mathbb{R} \rightarrow \mathbb{R}$ is a real function, $D$ is a diffusion coefficient, $N_{0}$ is a number of individuals at the initial time, and $\delta(x)$ is a Dirac function.

Thus, the classical solution to problem (12) exists and is unique for values of $x$ in bounded domains $U \subset \mathbb{R}$ and is given by

$$
u(x, t)=\frac{N_{0}}{\sqrt{(4 \pi D t)}} e^{-x^{2} / 4 D t} .
$$

If incorporate into (13) the parameters of reaction and advection will have a problem of the form

$$
\begin{gathered}
\frac{\partial u}{\partial t}(x, t)=D \Delta(u(x, t))+a \frac{\partial u}{\partial x}(x, t)+b u(x, t), \\
\text { in } x \in \mathbb{R}, \quad t \geq 0, \\
u(x, 0)=u_{0}(x),
\end{gathered}
$$

whose solution is given by

$$
u(x, t)=\frac{u_{0}}{\sqrt{(4 \pi D t)}} e^{-(x-a t)^{2} / 4 D t+b t} .
$$

If we consider $x \in \mathbb{R}^{n}$, then we have

$$
\begin{gathered}
\frac{\partial u}{\partial t}(x, t)=\Delta(u(x, t)), \quad x \in \mathbb{R}^{n}, t \geq 0, \\
u(x, 0)=g(x),
\end{gathered}
$$

where $u: U \subset \mathbb{R}^{n} \rightarrow \mathbb{R}, g: \mathbb{R}^{n} \rightarrow \mathbb{R}$ are real functions, with $U \subset \mathbb{R}^{n}$ being the solution to

$$
\varphi_{t}(x)=\frac{1}{(4 \pi D t)^{n / 2}} \int_{\mathbb{R}^{n}} e^{-\|x-y\|^{2} / 4 D t} g(y) d y .
$$

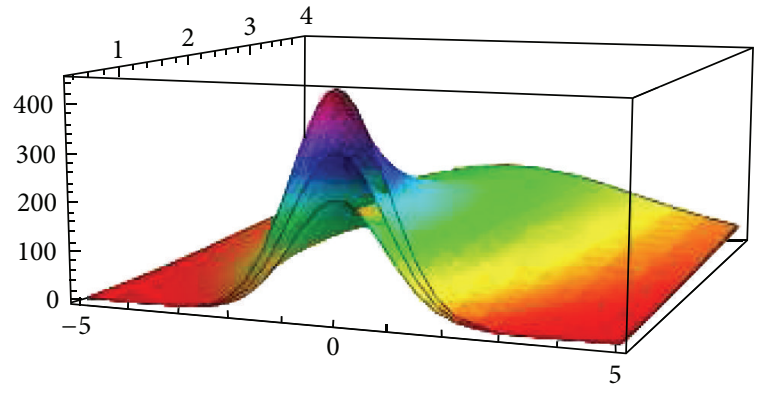

FIgURE 1: Graph of the fuzzy solution for $a=b=0$.

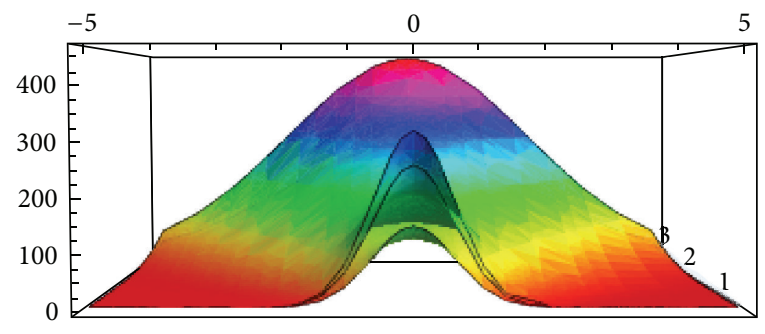

FIGURE 2: Graph of the fuzzy solution for $a=0$.

\section{Fuzzy Diffusion-Reaction- Advection Equation}

3.1. Fuzzy Solution in $\mathbb{R}$. Most of the phenomena involving diffusion are described by models that describe a particular dynamic in $\mathbb{R}$ and $\mathbb{R}^{2}$ and that is what will be done next. Consider a classical diffusion equation (15), where $D$ is a diffusion constant and $u_{0}$ is the number of individuals at the initial time.

Thus, we interpret a problem that works as follows. We know that the phenomenon occurs by diffusion, but its initial condition is not well determined. Thus, we can consider the initial condition as a fuzzy number and thereafter apply the principle of Zadeh's extension in initial condition. So we have the solution to the fuzzy initial value problems:

$$
\widehat{u}(x, t)=\frac{\widehat{u}_{0}}{\sqrt{(4 \pi D t)}} e^{-(x-a t)^{2} / 4 D t+b t},
$$

and their $\alpha$-levels are given by

$$
[u(x, t)]^{\alpha}=\frac{\left[u_{0}\right]^{\alpha}}{\sqrt{(4 \pi D t)}} e^{-(x-a t)^{2} / 4 D t+b t}
$$

which is plotted in Figures 1, 2, 3, and 10. An important note about these graphs is the fact that the deterministic solution for fuzzy initial value problem associated with the chart was kept deliberately fuzzy solution for the appropriate comparisons. About the $\alpha$-levels and their degree of membership a simulation of the evolution of the solution with time is made. A video also was available on https://www.youtube .com/watch? $\mathrm{v}=\mathrm{sHSfVfW67M4}$. The evolution of $\alpha$-levels is plotted in Figures 5, 6, 7, and 8 . 


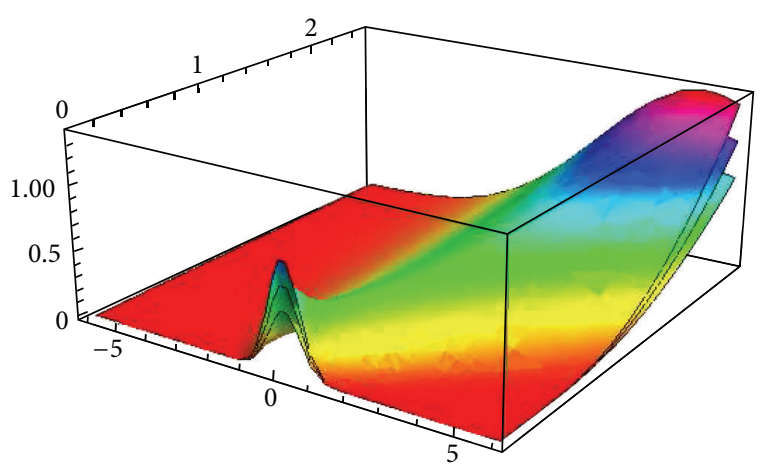

FIGURE 3: Graph of the fuzzy solution for $a, b \neq 0$.

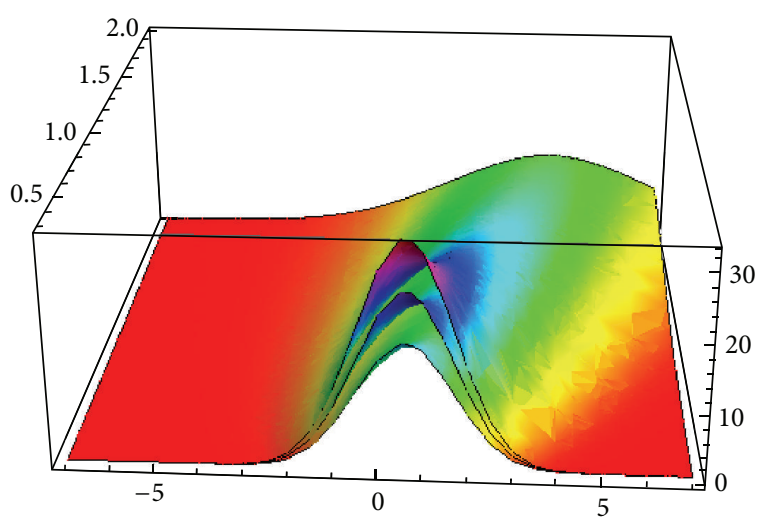

FIgURE 4: Graph of the fuzzy solution for $b=0$.

It is easily verified that there is stability and convergence to zero (see Figure 4) in cases where the process is described only by diffusion and advection. For this, note that

$$
\begin{aligned}
\lim _{t \rightarrow \infty} & \left(\operatorname{diam}\left(\left[\varphi_{t}\left(u_{0}\right)\right]^{\alpha}\right)\right) \\
& =\lim _{t \rightarrow \infty}\left(u_{01}^{\alpha}-u_{02}^{\alpha}\right) \frac{1}{\sqrt{4 \pi D t}} e^{-(x-a t)^{2} / 4 D t+b t} \\
& =\left(u_{01}^{\alpha}-u_{02}^{\alpha}\right) \lim _{t \rightarrow \infty} \frac{1}{\sqrt{4 \pi D t}} e^{-(x-a t)^{2} / 4 D t+b t} \\
& =\operatorname{diam}\left(\left[u_{0}\right]^{\alpha}\right) \lim _{t \rightarrow \infty} \frac{1}{\sqrt{4 \pi D t}} e^{-(x-a t)^{2} / 4 D t+b t}=0 .
\end{aligned}
$$

Thus, when time progresses we see that the fuzzy solution converges to the deterministic.

\section{Fuzzy Diffusion-Reaction- Advection Equation $\mathbb{R}^{2}$}

In two-dimensional case, we have the same behavior found in one-dimensional case. Of course, there is an inability to plot the graphs relating to two-dimensional fuzzy solution, but we can plot the graph of the spatial distribution for values of $t$ fixed, to make a more detailed study of these solutions. In

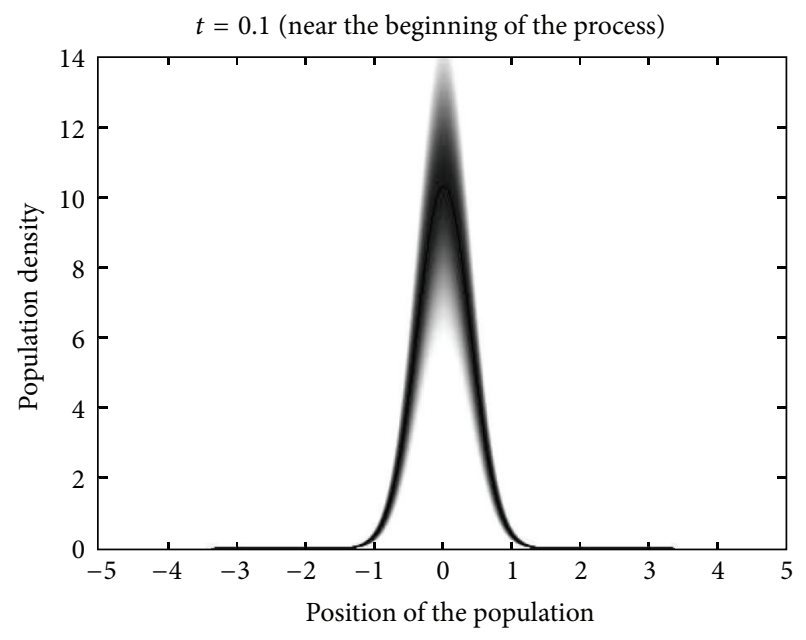

FIGURE 5: Dimensional graph of the fuzzy solution for $t=0.01$ involving only diffusion.

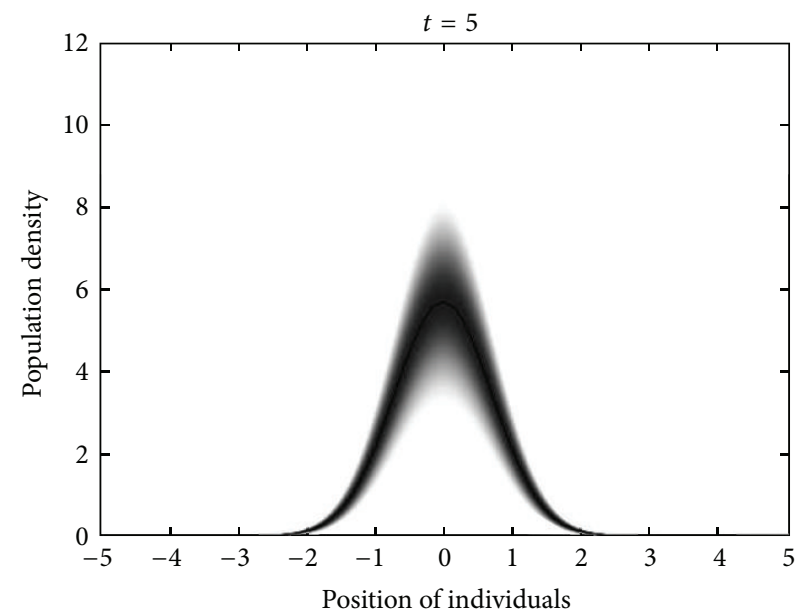

Figure 6: Dimensional graph of the fuzzy solution for $t=5$ involving only diffusion.

fact, we have that $\alpha$-levels of the two-dimensional solution to the fuzzy problem:

$$
\begin{gathered}
\frac{\partial u}{\partial t}(x, t)=D \Delta u(x, t)+\left(a \frac{\partial u}{\partial x_{1}}+c \frac{\partial u}{\partial x_{2}}\right)(x, t)+b u(x, t), \\
x \in \mathbb{R}^{2}, \quad t>0, \\
u(x, 0)=N_{0} \delta(x)
\end{gathered}
$$

which is given by

$$
[u(x, t)]^{\alpha}=\frac{\left[N_{0}\right]^{\alpha}}{4 \pi D t} e^{-\left(\left(x_{1}-a t\right)^{2}+\left(x_{2}-c t\right)^{2}\right) / 4 D t-b t},
$$

where $x=\left(x_{1}, x_{2}\right)$. So we can look at some cuts in fuzzy two-dimensional flow graph that give us an idea of fuzzy population distribution, that is, preserving uncertainty of the initial condition. 


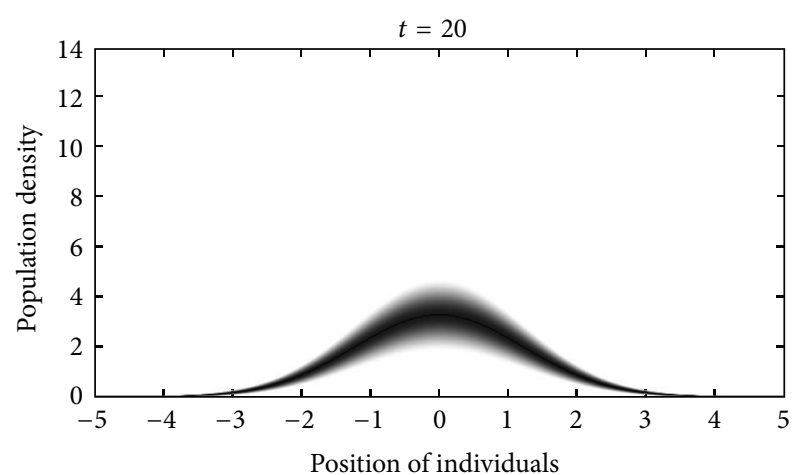

FIgURE 7: Dimensional graph of the fuzzy solution for $t=20$ involving only diffusion.

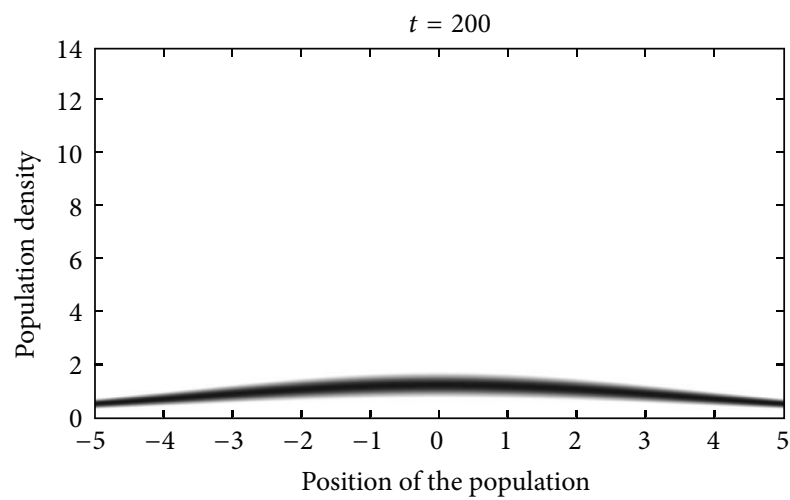

FIGURE 8: Dimensional graph of the fuzzy solution for $t=200$ involving only diffusion.

Just as in the one-dimensional case, we can observe the stability of fuzzy solution for this using the concept across, and this will be explored in the next section with dimension $n$.

\section{Stability and Uniqueness of the Diffusion Equation Fuzzy}

There is the possibility of defining a solution to the diffusion equation $n$ dimensional. Thus, we find significant results that generalize the properties of stability and unity of interest within the classical theory. To this end, consider the initial value problem given by equation

$$
\begin{gathered}
\frac{\partial u}{\partial t}(x, t)=f(u(x, t)), \quad x \in \mathbb{R}^{n}, t \geq 0, \\
u(x, 0)=g(x),
\end{gathered}
$$

where $u: U \subset \mathbb{R}^{n} \rightarrow \mathbb{R}, g: \mathbb{R}^{n} \rightarrow \mathbb{R}$ are a real functions, with $U \subset \mathbb{R}^{n}$. In this case, consider $f(u(x, t))=D \Delta u(x, t)$, which characterizes our diffusion equation.

In terms of models that simulate reality, we want to play the above problem as follows: we have knowledge of the law governing the growth of a certain variable, the equation partial differential diffusion, but the initial condition is not well determined and can be known only partially loading

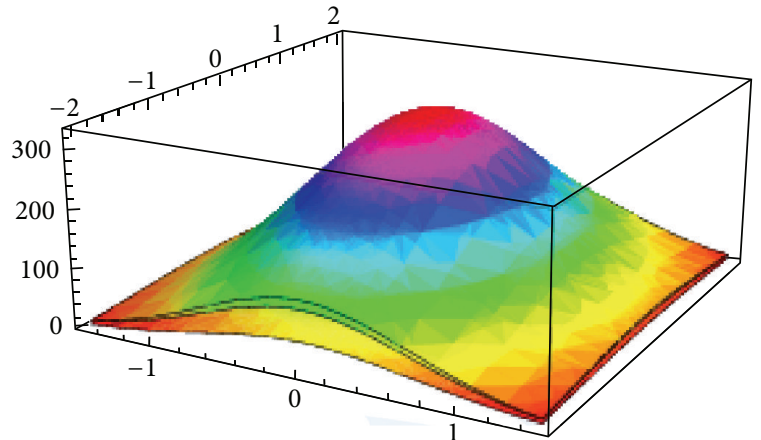

FIGURE 9: Fuzzy two-dimensional graph of the solution for $t=1$ involving advection-diffusion-reaction to $(a, c)=(0,0)$ and $b=1$.

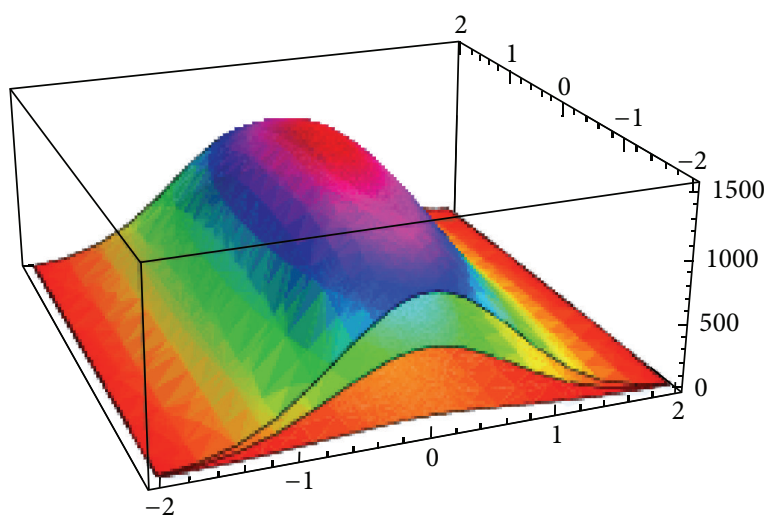

FIgURE 10: Fuzzy two-dimensional graph of the solution for $t=1$ involving advection-diffusion-reaction to $(a, c)=(0,0)$ and $b=1$.

with it a degree of uncertainty. Although the law is the classical solution to load the initial condition uncertainty over time, so let us consider the problem with initial condition uncertainty associated with the classical model given in (23):

$$
\begin{gathered}
\frac{\partial u}{\partial t}(x, t)=D \Delta u(x, t), \quad x \in \mathbb{R}^{n}, t \geq 0, \\
u(x, 0) \in \widehat{g}(x), \quad x \in \mathbb{R}^{n},
\end{gathered}
$$

where $\widehat{g}(x)$ is a fuzzy real function.

We know that when the function $g \in C\left(\mathbb{R}^{n}\right) \cap L^{\infty}(\mathbb{R})$, then this solution to the classical problem exists for all $x \in \mathbb{R}^{n}$ and $t \geq 0$ and is unique to values $x$ in limited domain $U \subset \mathbb{R}^{n}$. Thus we have the classical solution given by

$$
\varphi_{t}(x)=\frac{1}{(4 \pi D t)^{n / 2}} \int_{\mathbb{R}^{n}} e^{-\|x-y\|^{2} / 4 D t} g(y) d y .
$$

Thus, the solution of the problem is a continuous function in $x$. After we define Zadeh's extension of solution $\varphi_{t}(g(x))$ as Zadeh's extension of function $g(x) \rightarrow \varphi_{t}(g(x)) \rightarrow$ $\varphi(t, g(x))$, this solution will be denoted by $\widehat{\varphi}_{t}(g(x))$ and with

$$
\widehat{\varphi}_{t}(x)=\frac{1}{(4 \pi D t)^{n / 2}} \int_{\mathbb{R}^{n}} e^{-\|x-y\|^{2} / 4 D t} \widehat{g}(y) d y
$$


and the $\alpha$-levels are given by

$$
\begin{aligned}
{\left[\varphi_{t}(g(x))\right]^{\alpha} } & \\
= & {\left[\frac{1}{(4 \pi D t)^{n / 2}} \int_{\mathbb{R}^{n}} e^{-\|x-y\|^{2} / 4 D t} g_{1}^{\alpha}(y) d y,\right.} \\
& \left.\quad \frac{1}{(4 \pi D t)^{n / 2}} \int_{\mathbb{R}^{n}} e^{-\|x-y\|^{2} / 4 D t} g_{2}^{\alpha}(y) d y\right] .
\end{aligned}
$$

Then, we can define a fuzzy infinitely differentiable function with continuous derivatives of all orders as follows.

Definition 6. One says that a fuzzy function $\widehat{f}$ is $C^{\infty}$ when all $\alpha$-levels of $\widehat{f}$ are $C^{\infty}$.

Theorem 7. Suppose that $\hat{g} \in C(\mathbb{R}) \cap L^{\infty}\left(\mathbb{R}^{n}\right)$, and define $\widehat{u}$ as (26). Then

(i) $\widehat{u} \in C^{\infty}\left(\mathbb{R}^{n} \times(0, \infty)\right)$,

(ii) $\widehat{u}_{t}-D \Delta \widehat{u}(x, t)=0\left(x \in \mathbb{R}^{n}, t>0\right)$,

(iii) $\lim _{(x, t) \rightarrow\left(x^{0}, 0\right)} \widehat{u}(x, t)=\widehat{g}\left(x^{0}\right)$ for all $x^{0} \in \mathbb{R}^{n}$.

Proof. (i) The function $\left(1 /(D t)^{n / 2}\right) e^{-|x|^{2} / 4 D t}$ is infinitely differentiable with limited derivatives of all orders in $\mathbb{R}^{n} \times[\delta, \infty]$ for all $\delta>0$. Thus each $u_{i}^{\alpha} \in C^{\infty}\left(\mathbb{R}^{n} \times(0, \infty)\right)$ for each $i=\{1,2\}$. Then $\widehat{u} \in C^{\infty}\left(\mathbb{R}^{n} \times(0, \infty)\right)$.

(ii) Since $\widehat{u}=\left[u_{1}^{\alpha}, u_{2}^{\alpha}\right]$ and each $u_{i}^{\alpha}$ is a solution of diffusion equation, then $\widehat{u}$ solves problem (24).

(iii) Consider $\hat{g}=\left[g_{1}^{\alpha}, g_{2}^{\alpha}\right]$ where each $g_{i}^{\alpha}$ is initial condition to

$$
\begin{gathered}
\frac{\partial u}{\partial t}=D \Delta\left(u_{i}^{\alpha}(x, t)\right), \quad x \in \mathbb{R}^{n}, t \geq 0, \\
u_{i}^{\alpha}(x, 0) \in \widehat{g_{i}^{\alpha}}(x), \quad x \in \mathbb{R}^{n} .
\end{gathered}
$$

Thus fix $x^{0} \in \mathbb{R}^{n}, \epsilon>0$, and choose $\delta>0$ such that

$$
\left|g_{i}^{\alpha}(y)-g_{i}^{\alpha}\left(x^{0}\right)\right|<\epsilon \quad \text { se }\left|y-x^{0}\right|<\delta, y \in \mathbb{R}^{n}
$$

Then, if $\left|x-x^{0}\right|<\delta / 2$, we have

$$
\begin{aligned}
& \left|u_{i}^{\alpha}(x, t)-g_{i}^{\alpha}\left(x^{0}\right)\right| \\
& =\left|\int_{\mathbb{R}^{n}} \frac{1}{(4 \pi D t)^{n / 2}} e^{-|x-y|^{2} / 4 D t}\left[g_{i}^{\alpha}(y)-g_{i}^{\alpha}\left(x^{0}\right)\right]\right| d y \\
& \leq\left|\int_{B\left(x^{0}, \delta\right)} \frac{1}{(4 \pi D t)^{n / 2}} e^{-|x-y|^{2} / 4 D t}\left[g_{i}^{\alpha}(y)-g_{i}^{\alpha}\left(x^{0}\right)\right]\right| d y \\
& \quad+\left|\int_{\mathbb{R}^{n}-B\left(x^{0}, \delta\right)} \frac{1}{(4 \pi D t)^{n / 2}} e^{-|x-y|^{2} / 4 D t}\left[g_{i}^{\alpha}(y)-g_{i}^{\alpha}\left(x^{0}\right)\right]\right| d y \\
& =I+J .
\end{aligned}
$$

But, we have

$$
I \leq \epsilon \int_{\mathbb{R}^{n}} \frac{1}{(D t)^{n / 2}} e^{-|x-y|^{2} / 4 D t} d y=\epsilon
$$

by (29). If $\left|x-x^{0}\right| \leq \delta / 2$ and $\left|y-x^{0}\right| \geq \delta$, then

$$
\left|y-x^{0}\right| \leq|y-x|+\frac{\delta}{2} \leq|y-x|+\frac{1}{2}\left|y-x^{0}\right| \text {. }
$$

Then $|y-x| \geq(1 / 2)\left|y-x^{0}\right|$. Consequently

$$
\begin{aligned}
& J=2\left\|g_{i}^{\alpha}\right\|_{L_{\infty}} \int_{\mathbb{R}^{n}-B\left(x^{0}, \delta\right)} \frac{1}{(4 \pi D t)^{n / 2}} e^{-|x-y|^{2} / 4 D t} d y \\
& \leq \frac{C}{(D t)^{n / 2}} \int_{\mathbb{R}^{n}-B\left(x^{0}, \delta\right)} e^{-|x-y|^{2} / 4 D t} d y \\
& \leq \frac{C}{(D t)^{n / 2}} \int_{\mathbb{R}^{n}-B\left(x^{0}, \delta\right)} e^{-\left|x-x^{0}\right|^{2} / 16 D t} d y \\
&=\frac{C}{(D t)^{n / 2}} \int_{\delta}^{\infty} e^{-r^{2} / 16 t} d r \longrightarrow 0 \\
& \text { quando } t \longrightarrow 0^{+} .
\end{aligned}
$$

Then we have $\left|x-x^{0}\right|<\delta / 2$ and $t>0$; this way, we have $\left|u_{i}^{\alpha}(x, t)-g_{i}^{\alpha}\left(x^{0}\right)\right|<2 \epsilon$ for all $\alpha$-level and each $i=\{1,2\}$; then

$$
\left|x-x^{0}\right|<\frac{\delta}{2}, t>0 \quad \text { we have }\left|\widehat{u}(x, t)-\widehat{g}\left(x^{0}\right)\right|<2 \epsilon .
$$

Define

$$
K(x, t)= \begin{cases}\frac{1}{(4 \pi D t)^{n / 2}} e^{-|x|^{2} / 4 D t} & \text { if }\left(x \in \mathbb{R}^{n}, t>0\right) \\ 0 & \text { if }\left(\mathbb{R}^{n}, t<0\right) .\end{cases}
$$

Then we have that $K$ is a solution of the problem

$$
\begin{gathered}
\frac{\partial K}{\partial t}-\Delta K=0, \quad x \in \mathbb{R}^{n} \times(0, \infty), \\
K=\widehat{\delta}_{0}, \quad x \in \mathbb{R}^{n} \times\{t=0\},
\end{gathered}
$$

where $\delta_{0}$ denotes Dirac's measure in $\mathbb{R}^{n}$ per unit mass in point 0 .

Note that if $g_{i}^{\alpha}$ is bounded and continuous and $g_{i}^{\alpha}>0$ then

$$
\widehat{u}(x, t)=\frac{1}{(4 \pi D t)^{n / 2}} \int_{\mathbb{R}^{n}} e^{-\left(|x-y|^{2} / 4 D t\right) \hat{g}_{i}^{\alpha}(y)} d y
$$

is positive for all $\alpha$-level and for all $x \in \mathbb{R}^{2}$ and $t>0$.

Let's focus our attention to the nonhomogeneous initial value problem:

$$
\begin{gathered}
\frac{\partial u}{\partial t}-D \Delta u=f, \quad x \in \mathbb{R}^{n} \times(0, \infty), \\
u=\hat{g}, \quad x \in \mathbb{R}^{n} \times\{t=0\} .
\end{gathered}
$$

To solve this problem the following question must be answered: what is the formula to solve the following problem? Consider

$$
\begin{gathered}
\frac{\partial u}{\partial t}-D \Delta u=f, \quad x \in \mathbb{R}^{n} \times(0, \infty), \\
u=0, \quad x \in \mathbb{R}^{n} \times\{t=0\} .
\end{gathered}
$$


Consider the solution of the homogeneous problem. Note that the application $(x, t) \rightarrow K(x-y, t-s)$ is a solution of diffusion equation to $\left(y \in \mathbb{R}^{n}, 0<s<t\right)$ given. Now for $s$ fixed, the function

$$
u=u(x, t ; s)=\int_{\mathbb{R}^{n}} K(x-y, t-s) f(y, s) d y
$$

is solution of

$$
\begin{gathered}
\frac{\partial u}{\partial t}(x, t: s)-D \Delta u(x, t: s)=0, \quad x \in \mathbb{R}^{n} \times(s, \infty), \\
u(x, t ; s)=f(x, t: s), \quad x \in \mathbb{R}^{n} \times t=s
\end{gathered}
$$

which is solution of (23) to $t=s$ and $g=f$. Then $u(\cdot ; s)$ is not solution of (41). But by Duhamel principle, we can consider

$$
u(x, t)=\int_{0}^{t} u(x, t ; s) d s .
$$

Then, we have

$$
\begin{aligned}
u(x, t) & =\int_{0}^{t} \int_{\mathbb{R}^{n}} K(x-y, t-s) f(y, s) d y d s \\
& =\int_{0}^{t} \frac{1}{(4 \pi D(t-s))^{n / 2}} \int_{\mathbb{R}^{n}} e^{-|x-y|^{2} / 4(t-s)} f(y, s) d y d s
\end{aligned}
$$

to $x \in \mathbb{R}^{n}, t>0$. Now consider the following theorem.

Theorem 8. Define u given by (43) and $f \in C_{1}^{2}\left(\mathbb{R}^{n} \times[0, \infty)\right)$ with compact support. Then

(i) $u \in C_{1}^{2}\left(\mathbb{R}^{n} \times(0, \infty)\right)$,

(ii) $u_{t}(x, t)-D \Delta u(x, t)=f(x, t), x \in \mathbb{R}^{n}, t>0$,

(iii) $\lim _{(x, t) \rightarrow\left(x^{0}, 0\right)} u(x, t)=0$ for each point $x^{0} \in \mathbb{R}^{n}$.

Proof. (1) Since $K$ has a singularity at $(0,0)$, first we change variables to white

$$
u(x, t)=\int_{0}^{t} \int_{\mathbb{R}^{n}} K(y, s) f(x-y, t-s) d y d s .
$$

As $f \in C_{1}^{2}\left(\mathbb{R}^{n} \times[0, \infty)\right)$ has a compact support and $K=$ $k(y, s)$ is smooth near $s=t>0$, we compute

$$
\begin{aligned}
& u_{t}(x, t)= \int_{0}^{t} \int_{\mathbb{R}^{n}} K(y, s) f_{t}(x-y, t-s) d y d s \\
&+\int_{\mathbb{R}^{n}} K(y, t) f(x-y, 0) d y d s, \\
& \frac{\partial^{2} u}{\partial x_{i} \partial x_{j}}(x, t)= \int_{0}^{t} \int_{\mathbb{R}^{n}} K(y, s) \frac{\partial 2}{\partial x_{i} \partial x_{j}} f(x-y, t-s) d y d s, \\
&(i, j=1, \ldots, n) .
\end{aligned}
$$

Thus $u_{t}, D_{x}^{2} u, u, D_{x} u$ belong to $C\left(\mathbb{R}^{n} \times(0, \infty)\right)$.

(2) We then calculate

$$
\begin{aligned}
u_{t}(x, t)-D \Delta u(x, t) & \\
= & \int_{0}^{t} \int_{\mathbb{R}^{n}} K(y, s)\left[\left(\frac{\partial}{\partial t}-\Delta_{x}\right) f(x-y, t-s)\right] d y d s \\
& +\int_{\mathbb{R}^{n}} K(y, t) f(x-y, 0) d y \\
\leq & \int_{\epsilon}^{t} \int_{\mathbb{R}^{n}} K(y, s)\left[\left(-\frac{\partial}{\partial s}-\Delta_{y}\right) f(x-y, t-s)\right] d y d s \\
& +\int_{0}^{\epsilon} \int_{\mathbb{R}^{n}} K(y, s)\left[\left(-\frac{\partial}{\partial s}-\Delta_{y}\right) f(x-y, t-s)\right] d y d s \\
& +\int_{\mathbb{R}^{n}} K(y, t) f(x-y, 0) d y \\
:= & I_{\epsilon}+J_{\epsilon}+K .
\end{aligned}
$$

Now

$$
\left|J_{\epsilon}\right| \leq\left(\left\|f_{\epsilon}\right\|_{L^{\infty}}+\left\|D^{2} f\right\|_{L^{\infty}}\right) \int_{0}^{t} \int_{\mathbb{R}^{n}} K(y, s) d y d s \leq \epsilon C .
$$

Integrating by parts, we also find

$$
\begin{aligned}
I_{\epsilon}= & \int_{0}^{t} \int_{\mathbb{R}^{n}} K(y, s)\left[\left(\frac{\partial}{\partial t}-\Delta_{x}\right) f(x-y, t-s)\right] d y d s \\
& +\int_{\mathbb{R}^{n}} K(y, t) f(x-y, 0) d y \\
\leq & \int_{\epsilon}^{t} \int_{\mathbb{R}^{n}} K(y, s)\left[\left(\frac{\partial}{\partial s}-\Delta_{y}\right) f(x-y, t-s)\right] d y d s \\
& +\int_{\mathbb{R}^{n}} K(y, \epsilon) f(x-y, t-\epsilon) d y \\
& -\int_{\mathbb{R}^{n}} K(y, t) f(x-y, 0) d y \\
= & \int_{\mathbb{R}^{n}} K(y, \epsilon) f(x-y, t-\epsilon) d y-K,
\end{aligned}
$$

since $K$ solves the heat equation. We have

$$
\begin{aligned}
u_{t}(x, t)-D \Delta u(x, t) & =\lim _{\epsilon \rightarrow 0} \int_{0}^{t} \int_{\mathbb{R}^{n}} K(y, \epsilon) f(x-y, t-\epsilon) d y \\
& =f(x, t) \quad\left(x \in \mathbb{R}^{n}, t>0\right) .
\end{aligned}
$$


We can of course combine Theorems 7 and 8 to discover that $\widehat{u}$ is given by $\alpha$-levels in the form

$$
\begin{aligned}
{[\widehat{u}]^{\alpha}=[} & \int_{\mathbb{R}^{n}} K(x-y, t) g_{1}^{\alpha}(y) d y \\
& +\int_{0}^{t} \int_{\mathbb{R}^{n}} K(x-y, t-s) f(y, s) d y d s, \\
& \int_{\mathbb{R}^{n}} K(x-y, t) g_{1}^{\alpha}(y) d y \\
& \left.+\int_{0}^{t} \int_{\mathbb{R}^{n}} K(x-y, t-s) f(y, s) d y d s\right]^{\alpha} .
\end{aligned}
$$

Suppose that (50) is a fuzzy solution of the fuzzy initial value problem

$$
\begin{gathered}
u_{t}-D \Delta u=f, \quad \mathbb{R}^{n} \times(0, \infty), \\
u=\hat{g}, \quad \mathbb{R}^{n} \times t=0 .
\end{gathered}
$$

One way to study the behaviour of the $n$-dimensional fuzzy flow to the diffusion equation is using the concept of a diameter of a $\alpha$-level of a fuzzy number; to this, we will prove the following proposition.

Proposition 9. Define $\widehat{\varphi}_{t}(g(x))$ as a fuzzy flow given by (26) and $\left[\varphi_{t}(g(x))\right]^{\alpha}$ is of $\alpha$-level. Then,

$$
\begin{aligned}
& \sup _{x \in \mathbb{R}^{n}}\left(\operatorname{diam}\left(\left[\varphi_{t}(g(x)]^{\alpha}\right)\right)\right) \\
& \quad \leq \frac{1}{(4 \pi D t)^{n / 2}} \int_{\mathbb{R}^{n}}\left(\operatorname{diam}\left([g(y))^{\alpha}\right)\right) d y, \quad t>0 .
\end{aligned}
$$

In particular, $\lim _{t \rightarrow \infty} \operatorname{diam}\left(\left[\varphi_{t}(g(x)]^{\alpha}\right)\right)=0$, that is, $\operatorname{diam}\left(\left[\varphi_{t}(g(x)]^{\alpha}\right)\right)$, converges uniformly to 0 , when $t \rightarrow \infty$.

Proof. Since $\operatorname{diam}\left([g(y)]^{\alpha}\right)=g_{2}^{\alpha}(x)-g_{1}^{\alpha}(x)>0$, then we can define $f(x)=g_{2}(x)-g_{1}(x)$. Then we have proven that

$$
\sup _{x \in \mathbb{R}^{n}} \operatorname{diam}\left(\left[\varphi_{t}(g(x))\right]\right) \leq \frac{1}{(4 \pi D t)^{n / 2}} \int_{\mathbb{R}^{n}} f(y) d y, \quad t>0 .
$$

It is easy to see because $\operatorname{diam}\left(\left[\varphi_{t}(g(x))\right]^{\alpha}\right)=\varphi_{t}^{2}(g(x))-$ $\varphi_{t}^{1}(g(x))$.

Theorem 10. Let $U \subset \mathbb{R}^{n}$ be a bounded region; thus, if a problem of fuzzy initial condition is given by

$$
\begin{gathered}
\frac{\partial u}{\partial t}(x, t)=D \Delta u(x, t)+f(x), \quad x \in U \subset \mathbb{R}^{n}, t \geq 0, \\
u(x, 0) \in \widehat{g}(x), \quad U \times[t=0],
\end{gathered}
$$

where $g(x) \in C(U \times[t=0])$ and $f(x) \in C(U \times(0, \infty])$, then the fuzzy flow $\widehat{\varphi}_{t}(g(x))$ associated to problem (25) is unique for all fuzzy initial condition $\widehat{g}(x)$ given.
Proof. Let $u$ and $v$ be fuzzy flows associated to problem (26), then define $w=u-v$. Thus, we have that $w$ resolves the problem

$$
\begin{gathered}
\frac{\partial w}{\partial t}(x, t)=D \Delta w(x, t), \quad x \in U \subset \mathbb{R}^{n}, t \geq 0, \\
w(x, 0)=0, \quad U \times[t=0]
\end{gathered}
$$

which is a classical problem and, by the maximum principle, we have the maximum value of $w$ which is assumed at the border; then $w=0$ and $u=v$.

Now, we have an important tool in the study of uncertainties that involves the diffusion equation, because we know that that limited our domain conveniently; we have the unique solution to problems involving diffusion. Furthermore, the stability of this solution is guaranteed and classical functions retaining it is degree of uncertainty.

Theorem 11. Define $\widehat{u} \in C_{1}^{2}\left(\mathscr{F}\left(\mathbb{R}^{n} \times(0, T]\right)\right) \cap C\left(\mathscr{F}\left(\mathbb{R}^{n} \times\right.\right.$ $(0, T]))$ as a solution of problem

$$
\begin{gathered}
u_{t}-D \Delta u=0, \quad \mathbb{R}^{n} \times(0, T), \\
u=\widehat{g}, \quad \mathbb{R}^{n} \times\{t=0\}
\end{gathered}
$$

satisfying the estimate

$$
u_{i}^{\alpha}(x, t) \leq A e^{a|x|^{2}} \quad\left(x \in \mathbb{R}^{n}, 0 \leq t \leq T\right)
$$

for $A, a>0$ and $[\widehat{u}]^{\alpha}$. Then

$$
\sup _{\mathbb{R}^{n} \times[0, T]} \widehat{u}=\sup _{\mathbb{R}^{n}} \hat{g} .
$$

Proof. Suppose that

$$
4 a T<1 .
$$

Then we have

$$
4 a(T+\epsilon)<1
$$

for some $\epsilon>0$. Fix $y \in \mathbb{R}^{n}, \mu>0$, and define

$$
v_{i}^{\alpha}(x, t):=u_{i}^{\alpha}(x, t)-\frac{\mu}{(D(T+\epsilon-t))^{n / 2}} e^{|x-y|^{2} / 4(T-\epsilon-t) D} .
$$

Then we have

$$
\frac{\partial v_{i}^{\alpha}}{\partial t}-D \Delta v_{i}^{\alpha}=0, \quad \text { em } \mathbb{R}^{n} \times(0, T) .
$$

Fix $r>0$ and $U:=B^{0}(y, r), U_{T}=B^{0}(y, r) \times(0, T]$. By the theorem of the maximum

$$
\sup _{U_{T}} v_{i}^{\alpha}=\max _{\Gamma_{T}} v_{i}^{\alpha}
$$

where $\Gamma_{T}$ is a boundary of $U_{T}$. 
Now if $x \in \mathbb{R}^{n}$,

$$
\begin{aligned}
v_{i}^{\alpha}(x, 0) & =u_{i}^{\alpha}(x, 0)-\frac{\mu}{(D(T+\epsilon-t))^{n / 2}} e^{|x-y|^{2} / 4(T-\epsilon-t) D} \\
& \leq v_{i}^{\alpha}(x, 0)=g_{i}^{\alpha}(x, 0)
\end{aligned}
$$

and if $|x-y|=r, 0 \leq t \leq T$, then

$$
\begin{aligned}
v_{i}^{\alpha}(x, t) & =u_{i}^{\alpha}(x, t)-\frac{\mu}{(D(T+\epsilon-t))^{n / 2}} e^{|x-y|^{2} / 4(T-\epsilon-t) D} \\
& \leq A e^{a|x|^{2}}-\frac{\mu}{(D(T+\epsilon-t))^{n / 2}} e^{|x-y|^{2} / 4(T-\epsilon-t) D} \\
& \leq A e^{a(|y|+r)^{2}}-\frac{\mu}{(D(T+\epsilon))^{n / 2}} e^{|x-y|^{2} / 4(T-\epsilon) D} .
\end{aligned}
$$

Now, if $4 a(T+\epsilon)<1$ then $1 / 4(T+\epsilon)=a+\gamma$ for some $\gamma>0$. Then

$$
v_{i}^{\alpha}(x, t) \leq A e^{a(|y|+r)^{2}}-\mu(4(a+\gamma))^{n / 2} e^{(a+\gamma) r^{2}} \leq \sup _{\mathbb{R}^{n}} g_{i}^{\alpha}
$$

for $r$ selected sufficiently large. Thus,

$$
v_{i}^{\alpha}(y, t) \leq \sup _{\mathbb{R}^{n}} g_{i}^{\alpha}
$$

for all $y \in \mathbb{R}^{n}, 0 \leq t \leq T$ because $4 a T<1$. Let $\mu \rightarrow 0$, then $4 a T<1$ fails! We repeatedly apply the result above on the time intervals $\left[0, T_{1}\right],\left[T_{1}, 2 T_{1}\right]$, and so forth, for $T_{1}=1 / 8 a$.

Theorem 12. Let $\hat{g} \in C\left(\mathscr{F}\left(\mathbb{R}^{n}\right)\right), f \in C\left(\mathscr{F}\left(\mathbb{R}^{n} \times[0, T]\right)\right)$. Then there exists at most one solution $\widehat{u} \in C\left(\mathscr{F}\left(\mathbb{R}^{n} \times[0, T]\right)\right) \cap$ $C\left(\mathscr{F}\left(\mathbb{R}^{n} \times[0, T)\right)\right)$ of the initial-value problem

$$
\begin{gathered}
\frac{u_{i}^{\alpha}}{\partial t}-D \Delta u=f, \quad \mathbb{R}^{n} \times(0, T), \\
u=\widehat{g}, \quad \mathbb{R}^{n} \times\{t=0\}
\end{gathered}
$$

satisfying the growth estimate

$$
u_{i}^{\alpha}(x, t) \leq A e^{a|x|^{2}} \quad\left(x \in \mathbb{R}^{n}, 0 \leq t \leq T\right)
$$

for constants $A, a>0$.

Proof. If $\widehat{u}$ and $\widehat{v}$ satisfy (68) and (69), define $\widehat{w}:= \pm \widehat{u}-\widehat{v}$; we apply Theorem 11 to $\widehat{w}$ and we have the result.

\section{Conclusion}

The study of fuzzy diffusion equations brings an alternative method of treating process modeling where we have diffusive parameters and/or uncertain initial conditions. In the case of models that simulate reality, we have an inaccuracy in almost all parameters. This article develops an alternative method for treatment parameters which may have some uncertainty, for this, the fuzzy initial condition and setting conditions for the fuzzy solution is unique and ensures that these solutions are stable.

\section{Appendix}

In this work we have used the system of RGB colours for the graphical representation of the fuzzy solution of a fuzzy differential equation. In the RGB colour system, each colour is expressed by a combination of three values which have been considered here to be in the interval $[0,1]$. We may then consider each colour as an $\operatorname{RGB}(x, y, z)$ function with $x, y, z \in[0,1]$, where $\mathrm{RGB}(0,0,0)$ represents a black colour and $\operatorname{RGB}(1,1,1)$ represents a white colour.

Thus, given an $\alpha \in[0,1]$, the colour RGB $(1-\alpha, 1-\alpha, 1-\alpha)$ was used to colour the region in the plane bounded by the $\alpha$ level $[\mathbf{x}]^{\alpha}$; then the region bounded by $[\mathbf{x}]^{0}$ is coloured white, and if $\alpha=1$ then the region bounded by $[\mathbf{x}]^{1}$ is coloured black. Thus, the higher the degree of membership of a point $x$ is, the corker its colour will be.

In practice we partitioned

$$
0=\alpha_{0}<\alpha_{1}<\cdots<\alpha_{i}<\cdots<\alpha_{n}=1
$$

on $[0,1]$ and, for each $\alpha_{i}$, we used the equality $\left[\widehat{\varphi}_{t}\left(\mathbf{x}_{0}\right)\right]^{\alpha}=$ $\varphi_{t}\left(\left[\mathbf{x}_{0}\right]^{\alpha}\right)$ to determine the set $\left[\widehat{\varphi}_{t}\left(\mathbf{x}_{0}\right)\right]^{\alpha_{i}}$ as follows. The higher the value of $\alpha_{i}$, darker the color used to fill the region bounded by the set $\left[\widehat{\psi}_{t}\left(\mathbf{y}_{0}\right)\right]^{\alpha_{i}}$.

\section{Conflict of Interests}

The authors declare that there is no conflict of interests regarding the publication of this paper.

\section{Acknowledgment}

The authors express their gratitude to the Fundação de Amparo à Pesquisa de Mato Grosso, FAPEMAT (no. 332507/ 2012), for financial support of this research.

\section{References}

[1] L. A. Zadeh, "Fuzzy sets," Information and Computation, vol. 8, pp. 338-353, 1965.

[2] L. C. Barros and R. Bassanezi, Tópicos de Lógica Fuzzy e Biomatemática, IMECC-UNICAMP, Campinas, Brazil, 2010.

[3] P. Diamond, "Stability and periodicity in fuzzy differential equations," IEEE Transactions on Fuzzy Systems, vol. 8, no. 5, pp. 583-590, 2000.

[4] A. Kandel and W. J. Byatt, "Fuzzy differential equations," in Proceedings of the International Conference on Cybernetics and Society, Tokyo, Japan, October 1981.

[5] M. T. Malinowski, "On random fuzzy differential equations," Fuzzy Sets and Systems, vol. 160, no. 21, pp. 3152-3165, 2009.

[6] M. S. Cecconello, J. C. Leite, R. C. Bassanezi, and A. V. Brandão, "Periodic orbits for fuzzy flows," Fuzzy Sets and Systems, vol. 230, pp. 21-38, 2013.

[7] M. S. Cecconello, J. Leite, R. C. Bassanezi, and J. D. D. M. Silva, "About projections of solutions for fuzzy differential equations," Journal of Applied Mathematics, vol. 2013, Article ID 184950, 9 pages, 2013.

[8] J. D. Silva, J. Leite, R. C. Bassanezi, and M. S. Cecconello, "Stationary points-I: one-dimensional $p$-fuzzy dynamical systems," Journal of Applied Mathematics, vol. 2013, Article ID 495864, 11 pages, 2013. 
[9] M. S. Cecconello, J. C. Leite, R. C. Bassanezi, and A. J. V. Brandão, "On the stability of fuzzy dynamical systems," Fuzzy Sets and Systems, vol. 248, pp. 106-121, 2014.

[10] M. Oberguggenberger, "Fuzzy and weak solutions to differential equations," in Proceedings of the 10th International IPMU Conference, pp. 517-524, 2004.

[11] Y.-Y. Chen, Y.-T. Chang, and B.-S. Chen, "Fuzzy solutions to partial differential equations: adaptive approach," IEEE Transactions on Fuzzy Systems, vol. 17, no. 1, pp. 116-127, 2009.

[12] S. Seikkala, "On the fuzzy initial value problem," Fuzzy Sets and Systems, vol. 24, no. 3, pp. 319-330, 1987.

[13] O. Kaleva, "Fuzzy differential equations," Fuzzy Sets and Systems, vol. 24, no. 3, pp. 301-317, 1987.

[14] M. Oberguggenberger and S. Pittschmann, "Differential equations with fuzzy parameters," Mathematical and Computer Modelling of Dynamical Systems, vol. 5, no. 3, pp. 181-202, 1999.

[15] M. T. Mizukoshi, L. C. Barros, Y. Chalco-Cano, H. RománFlores, and R. C. Bassanezi, "Fuzzy differential equations and the extension principle," Information Sciences, vol. 177, no. 17, pp. 3627-3635, 2007.

[16] J. J. Buckley and T. Feuring, "Fuzzy differential equations," Fuzzy Sets and Systems, vol. 110, no. 1, pp. 43-54, 2000.

[17] H. Román-Flores, L. C. Barros, and R. C. Bassanezi, "A note on Zadeh's extensions," Fuzzy Sets and Systems, vol. 117, no. 3, pp. 327-331, 2001.

[18] O. Kaleva, "The Cauchy problem for fuzzy differential equations," Fuzzy Sets and Systems, vol. 35, no. 3, pp. 389-396, 1990.

[19] J. Leite, Sistemas dinâmicos fuzzy aplicados a processos difusivos [Ph.D. thesis], UNICAMP, São Paulo, Brazil, 2010. 


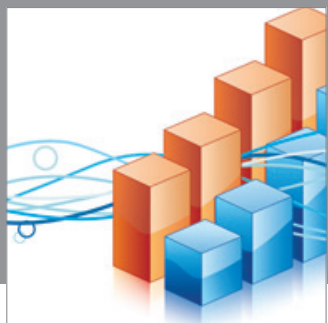

Advances in

Operations Research

mansans

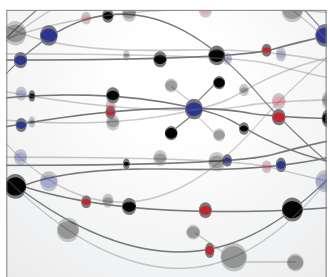

The Scientific World Journal
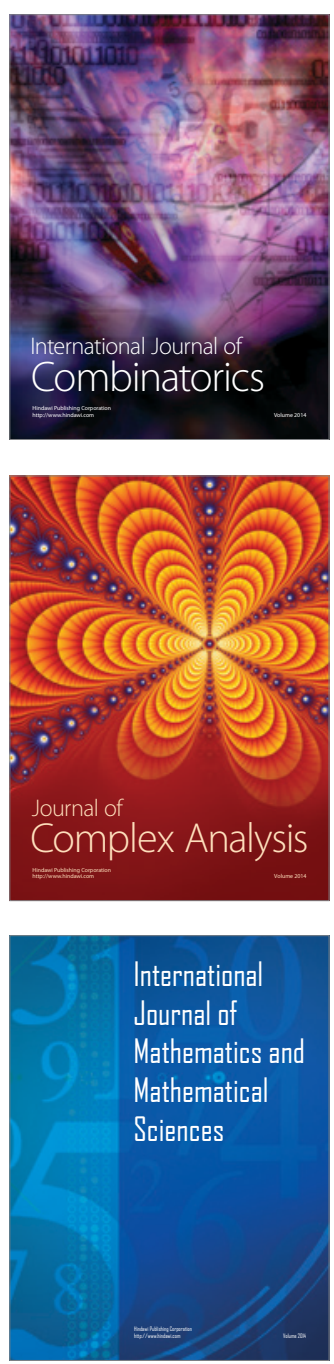
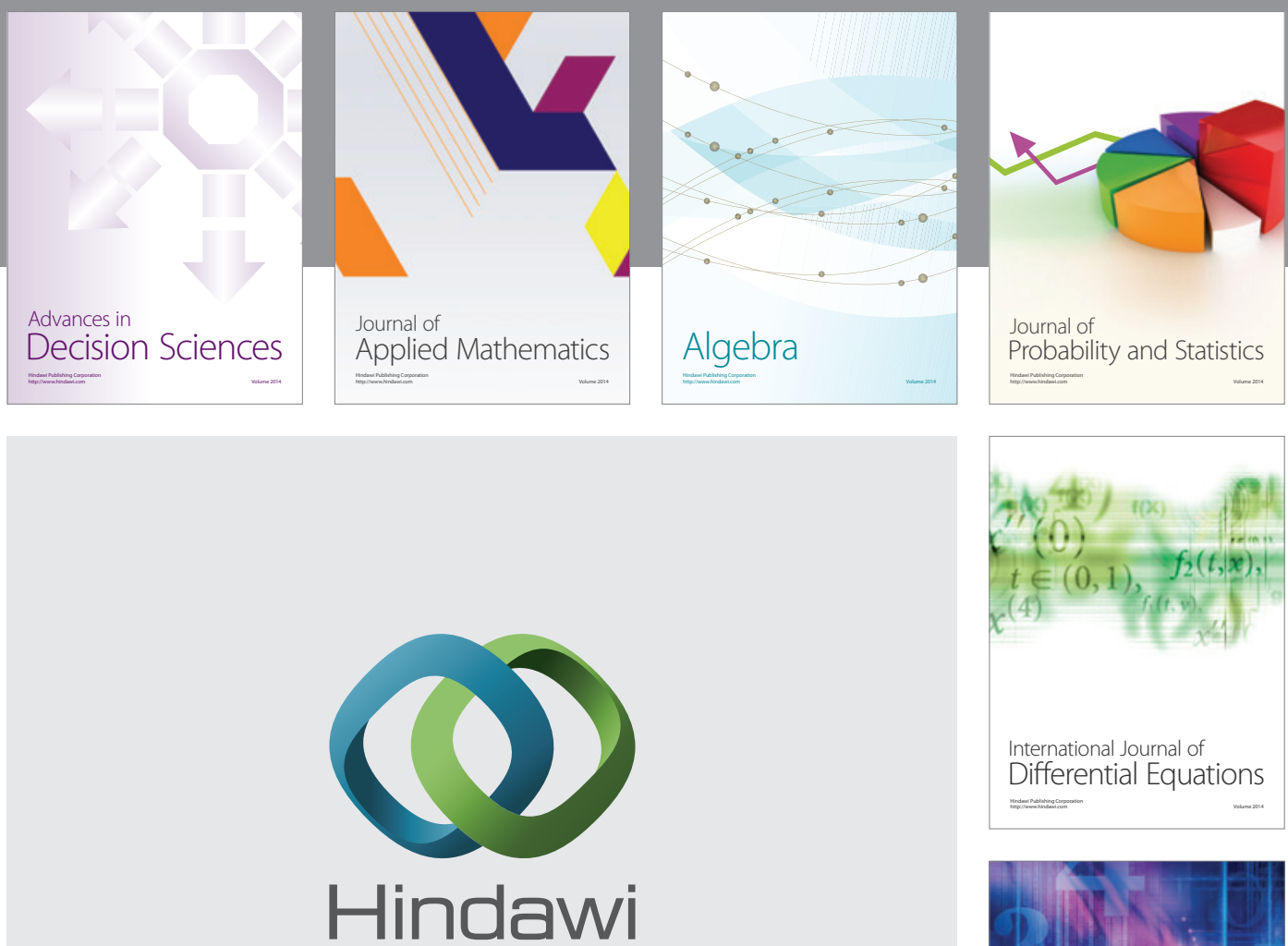

Submit your manuscripts at http://www.hindawi.com
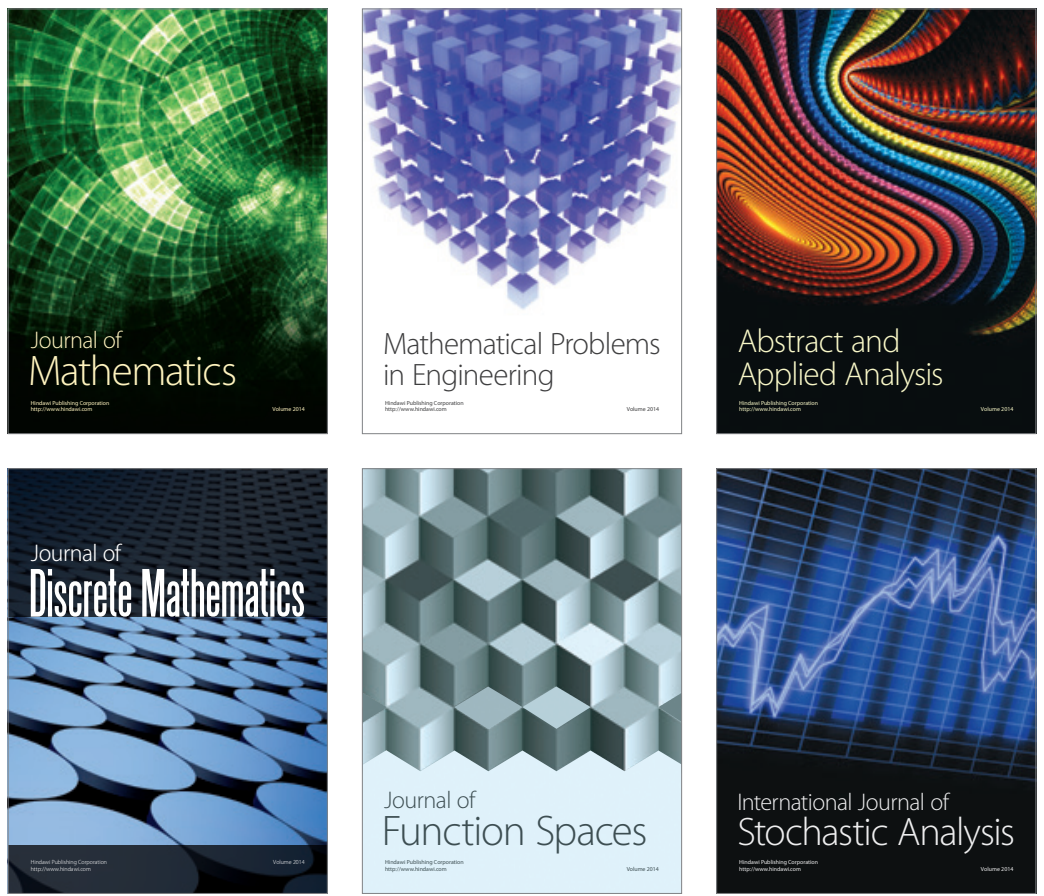

Journal of

Function Spaces

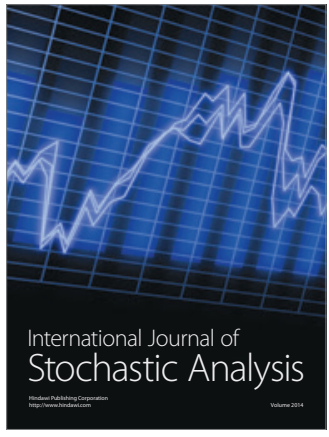

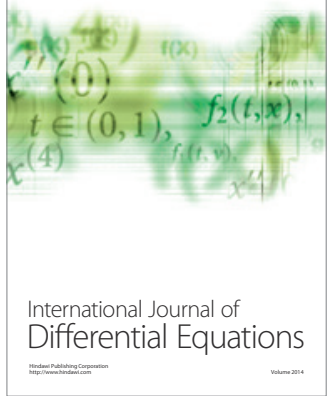
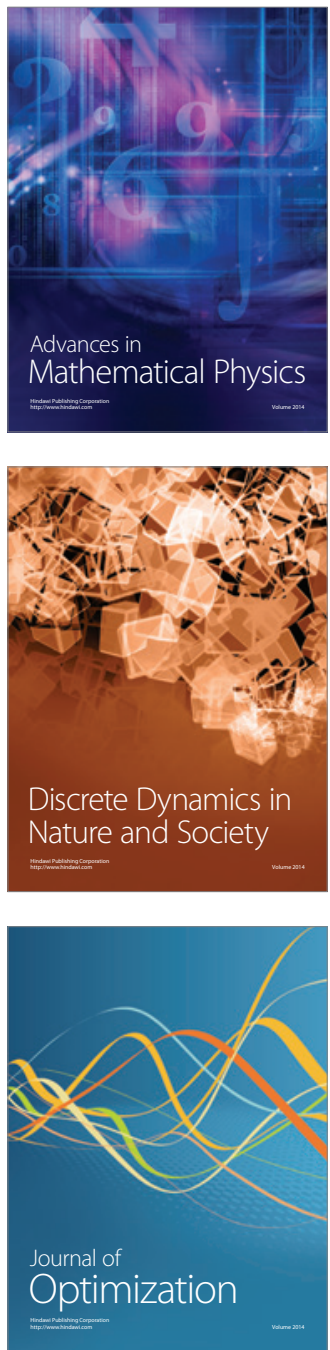